\title{
BEAT GESTURES MODULATE AUDITORY INTEGRATION IN SPEECH PERCEPTION
}

\author{
Emmanuel Biau ${ }^{1}$, Salvador Soto-Faraco ${ }^{1,2}$
}

\begin{abstract}
1 Departament de Tecnologies de la Informació i les Comunicacions, Universitat Pompeu Fabra, Barcelona, Spain

2 Institució Catalana de Recerca i Estudis Avançats (ICREA), Barcelona, Spain
\end{abstract}

\section{Corresponding author:}

Salvador Soto-Faraco

Departament de Tecnologies de la Informació i les Comunicacions

Universitat Pompeu Fabra

C. Roc Boronat, 138

08018 Barcelona, Spain

salvador.soto@icrea.es 


\section{ABSTRACT}

Spontaneous beat gestures are an integral part of the paralinguistic context during face-to-face conversations. Here we investigated the time course of beat-speech cointegration in natural speech perception conditions. We measured event-related potentials evoked by words pronounced with or without an accompanying beat gesture, while participants watched a spoken discourse. Words accompanied by a beat gesture elicited a positive shift in ERPs at early sensory (before 100ms) phase and at a later time window coinciding with the auditory component P2. As a control, there was no difference when participants only listened to the same speech passage without view of the speaker. We conclude that beat gestures are integrated with speech early on in time and modulate the sensory/phonological levels of processing. These results support the possible role of beats as a highlighter, helping the listener to direct the focus of attention on important information and modulate how verbal information is treated.

Keywords: speech processing; gestures; audiovisual integration; ERPs 


\section{1- INTRODUCTION}

Communicative, face to face interactions are amongst the most common and socially relevant behaviours in humans. In this context, visual information from the speaker's hand gestures has been shown to convey paralinguistic information useful for the comprehension of the spoken discourse. Indeed, studies using event-related potentials (ERPs) show that iconic gestures, whose shape depicts objects or actions and are related to semantic content of the message (McNeill, 1992), modulate the correlates of auditory speech processing in the perceiver. For example, Kelly, Kravitz \& Hopkins (2004) showed that iconic gestures modulate auditory integration of concurrent speech at early sensory/phonological stages as well as at late semantic stages, as a function of the semantic congruency between seen gesture and heard word. More recently, Kelly, Creigh \& Bartolotti (2010) have shown that iconic gestures are automatically integrated with speech despite being completely irrelevant for the task.

These examples highlight the relevance of paralinguistic hand movements, at least for gestures with a clear iconic meaning. However, the most common type of gestures by far in spoken behaviour, the beat gestures, has received much less attention in the literature (McNeill (1992), p.93: Frequency of gesture types). Beats are typically executed as rapid flicks of hand, have no semantic content of their own and are thought to play a role in the conception of the discourse organisation by being placed generally during the introduction of a new character or to highlight important point (McNeill, 1992). These gestures are pervasive in public addresses, such as political discourses, where for example, right-handed politicians tend to spontaneously present positive-value points with right-hand gestures whereas lefthanders use the left hand (Casasanto \& Jasmin, 2010). Using artificial materials in which speakers were explicitly instructed to emphasize intonation on target words, Krahmer \& Swerts (2007) found that producing beat hand gestures correlated with increased duration of high-frequency formants of the words they accompanied, thereby enhancing their acoustic prominence for the listeners. Thus, there is certain agreement in the literature that beat gestures reveal an intention from the speaker to put the stress on crucial points of the discourse.

However, the type of influence can this class of gestures exert on the listener's side during speech perception still remains largely unknown. One recent study has 
investigated how beat gestures can help the listener disambiguate sentences with a complex syntactic structure using ERPs (Holle et al., 2012). Holle et al. showed a modulation of the P600 component (indexing the difficulty of resolving complex syntactic structures) when beats emphasized which noun was the subject of the sentence. In the only fMRI study so far (as long as we are aware) to address beat gestures, Hubbard, Wilson, Callan \& Dapretto (2009) found stronger BOLD responses in high-order auditory areas (such as left STG/STS and the right planum temporale) when speech was accompanied by beats, compared to none-sense hand movements (like self-grooming). Moreover, STS responses were found only when beat gestures were presented with speech but not when presented on their own, reflecting a specific sensitivity of this brain area to gestures as part of the paralinguistic context. Hubbard et al.'s results suggest that beats and speech are parsed in an integrated fashion. Then, as beats can be considered as rapid and simple visual cues of the paralinguistic context, one can assume that they act early in speech processing, the same way lips modulate speech perception (Sumby \& Pollack, 1954; McGurk \& MacDonald, 1976). For example, van Wassenhove, Grant \& Poeppel (2005) showed that when lips movements are available, the early N1/P2 components of congruent speech integration are modulated, indexing the cointegration mechanisms of both modalities (Colin et al., 2002; Pilling, 2009). Like in the case of the sight of lip movements, beat gestures may exert some influence on how speech is processed and we expect to find some modulation on the early wordevoked ERPs reflecting the audiovisual integration mechanisms.

\section{Scope of the present study}

Maybe due to the absence of clear semantic contents in their shape or their high frequency of occurrence along the discourse, beat gestures processing in close-tonatural conditions of productions have received little attention. In order to shed some light on this question, we propose a different approach by presenting participants a real-life, recorded, TV broadcast of a political address in which the speaker (exformer Spanish president José Luís Rodríguez Zapatero) spontaneously gestured while speaking to the audience. EEG was recorded from the participants' scalp as they watched the discourse, and then we extracted event-related potentials (ERPs) time-locked to the onset of words synchronized with beat gestures, and compared them to ERP from equivalent words pronounced without accompanying gesture. In 
doing so, we investigated the time course of gesture-speech integration during perception of a running discourse, akin to how people experience speech in everyday life situations.

If beat gestures exert some influence on how speech is processed, we expect to find some modulation on the word-evoked ERPs. The latency of this possible modulation will be informative as to the level of processing at which gestures express their influence on speech.

\section{2- MATERIAL AND METHODS}

\subsection{Participants}

Twenty native Spanish speakers (12 females; mean age=23.8 years \pm 3.4 ) participated in the experiment after giving informed written consent. All participants were right-handed and had normal or corrected-to-normal vision. No one reported any known hearing deficit.

\subsection{Materials}

A political discourse of the former Spanish President Mr. José Luis Zapatero recorded in television conditions, served as experimental material (30/12/2010, "Balance económico de 2010", 17m and 46s duration, available at: http://www.youtube.com/watch?v=sOQ-pCjrd2g). In this video, the speaker stands behind a lectern, with the upper part of the body and head in full sight (hand/arms, chest and shoulders). We used video-editing software (Adobe Premiere Pro CS3) to select pairs of identical words within the discourse, but pronounced in two different contexts: synchronized with a beat gesture (Gesture Condition, GC) or without any accompanying hand gesture (No Gesture condition, NGC). For example, we selected two instances of the word "también", one synchronized with a rapid flick of the hand(s) and another pronounced with the hands of the speaker resting steady on the lectern, occurring at separate moments in the discourse (see fig. 1). In total, we could find 77 such pairs of words, applying criteria in line with the general definition of beat gestures described by McNeill (1992, pp15-16): rapid hand/finger flicks with two phases of movement and meaningless in itself. Beat gestures are also mostly localized in the peripheral gesture space. 
The onset time of each selected word (with and without beat gesture) was determined by visually and acoustically inspecting the spectrogram (using Praat v.5 software). Words with and without gesture were not different in average duration (respectively $0.498 \mathrm{~s} \pm 0.022$ and $0.464 \mathrm{~s} \pm 0.023 ; \mathrm{p}=0.675$ ) or intensity (respectively $69.28 \mathrm{~dB} \pm 0.58$ and $69.36 \mathrm{~dB} \pm 0.56 ; \mathrm{p}=0.917$ ). Their mean frequency of use was $632 \pm 1717$ (LEXESP frequency per million of words; measured with the software BuscaPalabras), and the mean length in letters was $6.4 \pm 2.3$. We did not limit the selection by syntactic category mostly because the scarcity of materials available (verbs:9; adverbs:5; pronouns:12; prepositions:4; nouns:32; adjectives:15). Nevertheless, we selected our beat gestures according to the previous description of McNeill (1992) which stipulates that beat gestures are not word specific but mark key moments and articulations in the narrative discourse.

In addition to word onsets, we also placed time marks at the onset of the gestures accompanying words, synchronized with the first video frame of the beat gesture (right when the preparation phase is initiated, see McNeill, 1992). On average, the gesture onset took place $200 \pm 102 \mathrm{~ms}$ before the acoustic onset of the corresponding word. For the words pronounced without gesture, the time stamp was placed at the exact point in time from acoustic onset as its homologue with gesture. Videos were edited using Adobe Premiere Pro CS3 and saved in AVI format using 5.1 sound systems. This allowed us to use one of the audio channels to place the time triggers for the ERP and therefore ensure precise synchrony between video, sound, and triggers during the stimulus presentation and EEG recording. We edited two versions of Mr. Zapatero's address: AudioVisual (AV henceforth, in which video and sound were presented) and Audio alone modality (A henceforth, in which only the sound was presented, with a central fixation cross on a blank display).

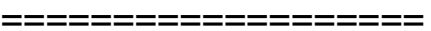

Please insert Figure 1 about here 


\subsection{Experimental Procedure}

Participants were individually presented with the two versions of the spoken discourse (AV and A modalities, order counterbalanced across participants). The room was sound attenuated and electrically shielded. During the presentation, participants' EEG was measured (see details below). Sound was delivered through headphones. Before the experiment, participants were told they would be given a memory test after each video about the content of the speech so that they had to listen carefully (10 item questionnaires were used after each video clip). Each item of the questionnaire consisted of 2 propositions of which participants had to select the one that had appeared in the discourse. For each memory test, participants were given 3 minutes to answer. This memory task was included to promote an attentive state as the participants watched the materials. After participants had read the instructions, a central fixation cross $(5 \mathrm{~s})$ on the screen gave way to the first video clip, with four one-minute resting breaks inserted at regular intervals. When the first video finished, participants had 3 minutes to complete the memory questionnaire. Then, after a pause of 5 minutes, the second version of the video began, followed by the second memory questionnaire.

\subsection{Electrophysiological recording}

Electrophysiological data were recorded (Brain Vision Recorder 1.05; Brain Products) at a rate of $250 \mathrm{~Hz}$ from 31 tin electrodes placed according to the 10-20 convention. An electrode placed at the participant's tip of the nose was used as reference but a re-referenciation was applied using the linked mastoids. Impedances were kept below $3 \mathrm{k} \Omega$. EEG activity was filtered off-line using a $30 \mathrm{~Hz}$ high-pass filter and a $0.05 \mathrm{~Hz}$ low-pass filter ( $24 \mathrm{~dB} /$ octave). Eye blink artefacts were corrected using the procedure of Gratton \& Coles (1989). The remaining artefacts (amplitude changes greater than $70 \mu \mathrm{V}$ within 200ms) were removed applying an automatic inspection on raw EEG data. Epochs ranged from -100 to $500 \mathrm{~ms}$ with respect to word onset. Baseline correction was performed in reference to pre-stimulus activity $(100 \mathrm{~ms})$ and individual averages were digitally re-referenced to the mastoid. In each condition, the grand average was obtained by averaging individual average waves. For the ERP analysis we used clusters of five electrodes centred on median sites at four scalp Regions: Frontal ( $F z, F 3, F 4, F C 1, F C 2)$, Central $(C z, C 3, C 4, C P 1, C P 2)$, Parietal 
(Pz, P3, P4, PO1, PO2) and Occipital (Oz, O1, O2, PO1, PO2). The mean amplitude of the ERP to the (auditory) words was calculated for each participant.

\subsection{Analysis}

\subsubsection{AV modality}

We first look at the ERPs in the AV modality, the condition of interest, where the sight of beats and the sound of speech were available. ERPs corresponding to words with and without gesture in the AV modality were first compared using point-by-point paired $t$-tests. We compared the whole signal between conditions, each $2 \mathrm{~ms}$, from 100 to $500 \mathrm{~ms}$ post onset. This difference wave analysis served to have a first overall view of the differences between conditions, given the lack of strong a priori hypothesis about timing or distribution of effects. This analytical approach helped us to select time windows of interest to analyse later with corrected ANOVAs. In this prospective point-by-point analysis the significance criterion was set at $\mathrm{p}$-level $<0.05$ for at least 20 consecutive ms (i..e, 10 samples). Following Guthrie \& Buchwald, (1991), we run 1000 simulations of data from two conditions in 20 participants (500 $\mathrm{ms}$ at $512 \mathrm{~Hz}$ sampling rate, random series with autocorrelation $\mathrm{r}=.85$ ) and found out that the probability of 10 consecutive significant samples at $p<.05$ (two tailed) was $p=.024$. Therefore, our criterion was well within the acceptable level of Type I error.

Then, for each the time windows determined with t-tests, the mean amplitudes of ERPs were submitted to a $2 \times 4$ repeated-measures analysis of variance (ANOVA) with the factors gesture (gesture versus no gesture), scalp region (clusters Frontal, Central, Parietal and Occipital) and Order of presentation (AV first or after A). Greenhouse-Geisser correction was applied in ANOVAs to control for sphericity violation.

\subsubsection{Audio modality}

We applied the same approach of analysis with the ERPs recorded during the audio modality. For comparison, the ANOVAs were applied on the time windows determined previously on ERPs from AV modality, so that the modulation due to adding visual information could be logically interpreted. First, this condition allowed us to control the validity of ERPs evoked by words embedded in a natural continuous speech discourse. Indeed, in our paradigm it was essential to ensure that classical ERPs components reflecting auditory integration were identifiable within this context. 
Previous literature has shown that auditory integration is reflected by two main ERP components situated at around $100 \mathrm{~ms}$ and $200 \mathrm{~ms}$ from word onset, respectively N1 and P2 components (e.g., Näätänen et al., 1997, see also van Wassenhove, Grant \& Poeppel, 2005; Stekelenburg \& Vroomen, 2007 using audio-visual stimuli for examples). If these components are identifiable in the A modality, it supports the validity of using a continuous speech and that word onsets were salient enough to evoke a detectable ERP response. Second, contrasting the word pronounced with gesture vs. a different token of the same word pronounced no gesture in the audiomodality alone allowed us to control for effects due to possible acoustic differences between the two exemplars of the word (i.e., in this condition the video was not available).

\subsubsection{AV-A subtraction}

In order to further single out the possible contribution of gestures to spoken word processing independent of possible acoustic differences between the two instances of each word, we also analysed the epoch signal and mean amplitude difference AV$A$, in both gesture conditions $\left((A \bigvee-A)_{\text {gesture }}\right.$ and $\left.(A \bigvee-A)_{\text {no gesture }}\right)$. This subtraction should remove any effect directly explained by possible differences between acoustic realizations of the two instances of each word in a pair and single out the effect of visual or audiovisual interaction on speech processing. We applied the same approach of analysis described above.

\subsubsection{Visual gesture analysis}

Finally, ERP data time locked to the visual onset of gestures were also analysed the same. In this case, time triggers were time-locked to gesture onsets. This extra analysis allowed us to have additional temporal information of gesture processing, instead of looking at the auditory integration alone.

\section{3- RESULTS}

\subsection{Memory questionnaire}

Participants achieved a correct response rate of $80.22 \pm 9.82 \%$, which was clearly above chance $\left(X^{2}(21)=88.5 ; p<0.0001\right)$. This suggests that participants attended carefully to the stimuli during the presentation. We also ran a t-test between scores 
obtained after either an AV or A modality of presentation. Results revealed no difference in performances according to the modality of discourse presentation $(t(38)<0.001 ; p>1)$. This suggests that memory for the verbal information in our paradigm was similar with and without visual support.

\subsection{ERPs to words: Audio-Visual modality}

Point by point paired $t$-tests between gesture and no gesture words in the audiovisual condition reflected significant differences within two time windows: early at [10-36ms] and late at [220-280ms] (see fig. 2).

Please insert Figure 2 about here

The ANOVAs including the factors Gesture (with and without), scalp Region (clusters Frontal, Central, Parietal and Occipital) and Order of presentation revealed differences between the gesture and no gesture conditions in both time windows: the mean amplitude of words with gesture was significantly more positive than that of words without in the early window [10-36ms], $(F(1,18)=13.07 ; p=0.002)$; and the late window [220-280ms] $(F(1,18)=8.05 ; p=0.011)$. A main effect of Region was found in the late window only $(F(3,54)=5.51 ; p=0.023)$. An interaction between Gesture and Region was found in the early window $(F(3,54)=3.64 ; p=0.043)$ and revealed a greater difference between conditions at the centro-parietal cluster $\mathrm{Cz}$ $(F(1,19)=21.55 ; \quad p=0.002)$ than at frontal $(F(1,19)=22.59 ; \quad p=0.002)$, parietal $(F(1,19)=6.58 ; p=0.019)$ or occipital $(F(1,19)=1.91 ; p=0.18)$ clusters. The effect of Gesture was significant in all four clusters but the difference between ERPs elicited by words with gesture and words without gesture was larger at the parietal cluster $(F(1,19=10.79 ; p=0.004)$ than at frontal $(F(1,19=6.84 ; p=0.017)$, central $(F(1,19=8.34$; $\mathrm{p}=0.009)$ or occipital $(\mathrm{F}(1,19=9.18 ; \mathrm{p}=0.007)$ clusters. We found no interaction between Condition and Order neither in the early $(F(1,18)=0.29 ; p=0.6)$ nor late $(F(1,18)=0.04 ; p=0.85)$ time windows. Then, we tested the lateralisation by grouping all the left electrodes from the 4 clusters separately to the equivalent right clusters. We applied a repeated-measure ANOVA, with factors Condition and Hemisphere. 
We found no interaction between Condition and Hemisphere in both time windows (respectively, $F(1,19)=1.80 ; p=0.20$ and $F(1,19)=1.71 ; p=0.21$ ).

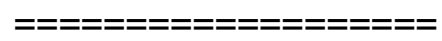

Please insert Figure 3 about here

The results from the Audio-Visual modality suggest that auditory speech integration is modulated by the co-occurring gesture at early stages (before $300 \mathrm{~ms}$ post onset) when visual information of the speaker is available (see fig. 3). Yet, before accepting this interpretation and deriving the possible implications, some further analyses are necessary.

\subsection{ERPs to words: Auditory modality}

Point by point paired $t$-tests comparing ERPs to words with and without gestures reflected significant differences according to the significance criterion of 20 consecutive significant ms. We only found differences for an early [10-36ms] time window (see fig. 2). The ANOVAs (one for each of the predefined time windows in AV modality) including the factors Gesture (with and without) and scalp Region (clusters Frontal, Central, Parietal and Occipital) revealed differences between the gesture and no gesture conditions only in the early time window $[10-36 \mathrm{~ms}](F(1,18)=4.84$; $\mathrm{p}=0.041$ ), where the mean amplitude of words with gesture was significantly more negative than that of words without (note that the polarity of this difference is opposite to the significant difference found in the AV modality in this time window). There was no significant difference between mean amplitudes of words with gesture and words without in the late $[220-280 \mathrm{~ms}]$ time window $(F(1,18)=0.01 ; p=0.928)$. An effect of Region was found only in the late window, but no interaction Gesture $x$ Region was found in either time window (see table 1). We found no interaction between Condition and Order neither in the early $(F(1,18)=0.84 ; p=0.372)$ nor late $(F(1,18)=1.36 ; p=0.259)$ time windows. We applied a repeated-measure ANOVA, with factors Condition and Hemisphere. We found no interaction between Condition and Hemisphere in both time windows (respectively $F(1,19)<1 ; p=0.977$ and $F(1,19)<$ $1 ; p=0.958)$. 
Regardless of the analysis of gesture vs. no gesture differences, it is worth noting that the auditory potentials evoked by the words contained the classical ERP components of auditory integration, despite the words were embedded in a continuous, naturally spoken speech discourse. Indeed, the N1-P2 complex reflecting sensory/phonological processing in auditory integration had a topographic distribution well in line with previous studies: N1 maximal at fronto-central sites (peak latency at $124 \mathrm{~ms}$ and $120 \mathrm{~ms}$ respectively for $\mathrm{G}$ and $\mathrm{NG}$ condition at $\mathrm{Cz}$ ) and $\mathrm{P} 2$ bilateraltemporal (peak latency at $180 \mathrm{~ms}$ and $174 \mathrm{~ms}$ respectively for $\mathrm{G}$ and $\mathrm{NG}$ condition at $\mathrm{Cz}$, see fig. 4). Interestingly, even if there is effectively a difference between mean amplitudes of words with gesture and without, classical ERP components of auditory integration are not directly affected because the modulation is found earlier.

In summary, in the absence of the visual co-speech information, typical ERP components reflecting auditory-only word processing are equivalent across words pronounced with or without beat gestures. This shows that in our A control modality, integration of both types of words is globally the same. Remarkably, we detected a difference between conditions at very early stages (early time window), perhaps due to some acoustic differences arising from the pronunciation of the words with and without the gesture (Krahmer \& Swerts, 2007). Importantly, this difference had an inverse polarity compared with the difference found in the analysis of $A V$ modality (above). In the subsequent analysis, this interaction is dealt with.

\subsection{ERPs to words: AV-A subtraction}

The previous analyses revealed differences in processing between words with gesture vs. without gesture for the AV modality which were either absent ([220$280 \mathrm{~ms}])$ or inverted in polarity $([10-36 \mathrm{~ms}])$ when processing these same words in the A modality. This pattern suggests a specific role of seeing beat gestures on the auditory processing of words. In order to support this interpretation, we ran a new round of analyses on the AV - A subtraction signal, so that any possible acoustic 
differences between the two instances of the target words could be effectively cancelled out. Point by point paired $t$-tests revealed again an early significant time window at [10-36ms] post-stimulus. The significant later time window determined in the AV modality was only partially visible (see fig. 2).

We analysed the average voltage differences in the same pre-defined time windows (early and late) with the same factors as in the previous analyses (Gesture and Scalp Region). The ANOVAs confirmed a modulation at early stages of speech processing. Indeed, in the early [10-36ms] time window, the ERP difference signal for words synchronized with gestures was significantly more positive than for words without gesture $(F(1,18)=11.55 ; p=0.003)$. Again, this polarity confirms the significant difference found in the analysis of the AV modality in this time window, and is opposite to the one found in the A modality. For the late time window [220-280ms], the ANOVA revealed nearly significant differences between the two gesture conditions $(F(1,18)=4.09 ; p=0.058)$. We found no interaction between Condition and Hemisphere in both time windows (respectively $F(1,19)=0.88 ; p=0.361$ and $F(1,19)=0.86 ; p=0.364)$.

As we found a tendency of effect of Condition in the late time window, we decided to run individual one-way repeated-measures ANOVAs (with the factor Gesture) in order to confirm the reliability of the main effect of Gesture within each scalp region separately. We found a significant positive shift of Gesture ERPs in the parietal cluster $(F(1,19)=4.53 ; p=0.046)$. For the remaining clusters, the same trend was detected albeit only at marginally significant levels ( $F z: F(1,19)=3.20 ; p=0.089$, $C z: F(1,19)=3.62 ; p=0.072$, Oz: $F(1,19)=3.68 ; p=0.07)$. See fig. 3 .

Looking at the peak of the early time window difference $(\mathrm{Cz}$, peak latency at $23 \mathrm{~ms}$ after stimulus onset), topographical distributions of the subtraction signal (AV$A)_{\text {gesture }}-(A V-A)_{\text {no gesture }}$ show a mean difference of profile between conditions at left centro-parietal sites. This pattern is still true in the late time window, although the differences are visually less clear $(\mathrm{Cz}$, peak latency at $273 \mathrm{~ms}$ after stimulus onset, see fig. 5).

Please insert Figure 5 about here 
In summary, when ERPs to the audiovisual words are normalized by ERPs to auditory words, results confirm that co-speech beat gestures modulate the auditory evoked potential to words, reflecting the effect of the sight of gestures on early stages of speech processing. Because the Av signal was normalized by the A signal, this result cannot be entirely explained by acoustic feature differences between word tokens. This modulation is likely to occur prior to semantic processing (estimated to occur at around 400ms post stimulus, see for example Wu \& Coulson, 2007).

\subsection{ERPs to visual gestures}

In order to gather additional information on the time course of integration between the beat gesture and the accompanying words, we compared the ERPs evoked by the gestures (i.e., time locked with gesture onsets at the first video frame of the cospeech gesture) to the equivalent point in time with respect to the onset of the corresponding word in the no-gesture condition. We analysed the time window, determined on the average signal: [344-366ms] post stimulus (see fig. 2). The ANOVA, including the factors Gesture (with and without) and Scalp Region (clusters Frontal, Central, Parietal and Occipital), showed an effect of Gesture $(F(1,19)=4.64$; $p=0.044)$ but not of Region $(F(3,57)=0.80 ; p=0.496)$. However, there was an interaction Condition by Region $(F(3,57)=12.78 ; p<0.001)$. A post-hoc paired t-test showed a Condition effect for clusters $F z$ and $C z$ only (respectively $F(1,19)=10.74$; $p=0.004$ and $F(1,19)=11.28 ; p=0.003)$. We found no interaction between Condition and Hemisphere $(F(1,19)<0.01 ; p=0.99)$.

These results suggest that integration of beat gestures occurs after the acoustic onset of synchronized words. Thus, it suggests that the beat and the accompanied word are integrated in two respective overlapping time windows (see fig. 6).

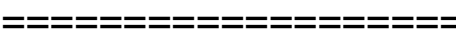

Please insert Figure 6 about here 


\section{4- DISCUSSION}

Beat gestures that speakers so frequently use to accompany their utterances constitute a very prominent part of the paralinguistic context in which listeners perceive spoken messages. Yet, the role of these gestures in comprehension has been seldom addressed. Here we aimed at investigating the neural correlates of beat-speech online integration during a continuous discourse perception, in a naturalistic situation. We found that the sight of beat gestures synchronized with speech modulated auditory integration mainly at two stages. At an early stage [10$36 \mathrm{~ms}]$, as well as at a later time window [220-280ms] coinciding, in time, with the P2 classical component. In both cases, gestures elicited a positive shift in word-evoked ERPs compared to the same words uttered without gestures within the same discourse. The P2 is a component which is often regarded as a robust signature of auditory processing in speech perception (Näätänen, 2001; van Wassenhove, Grant \& Poeppel, 2005). This component, or at least, the time window where it occurs (150ms-250ms) is thought to be modulated by attentional manipulations (Hillyard, Hink, Schwent \& Picton 1973; Picton \& Hillyard, 1974), and it also can be subject to cross-modal modulation by vision (Stekelenburg \& Vroomen, 2007).

The early [10-36ms] and late [220-280ms] modulations of the spoken signal found here cannot be attributed to a misplacing of ERP triggers because the recordings of the auditory modality allowed to correctly identifying N1/P2 components at the right timings and with a classic topographical distribution on the scalp in comparison with prior literature. When we subtracted any effect due to a possible acoustic difference in word pronunciation, using the AV-A difference wave, the early positive shift remained significant as in the direct analysis of the AV signal. The late modulation by gesture found in AV remained true in the subtraction analysis, albeit constrained mainly to parietal sites. Interestingly, the comparison between ERPs to words with and without gesture resulted very different when visual information was 
not available, in the Audio alone modality. There was no difference in the late window [220-260ms], and the difference at the early time window [10-36ms] had the opposite polarity (i.e., negative shift). Then, by looking at ERPs time locked to gesture onsets, we found that beat gestures seemed to be integrated in a time window [340-370ms] which overlaps with the auditory integration timing of synchronized words in our paradigm.

The temporal alignment between beat and speech allows us to assume that gestures modulated the prior visuospatial context in which the ensuing word occurred. The relative precocity of the two gesture-based modulations on the speech signal can be explained by the fact that beat gestures were initiated before the word onset (200 ms \pm 101.9 on average). First, on the video, it was effectively possible to observe that the hand gesture was initiated before the targeted word was pronounced. In consequence, the sight of the beat was available to the listeners well in advance of the sound of the word. Second, ERPs time locked with gesture onsets revealed that the evoked potential reflecting the processing of beats has a latency window of [340-370ms] post stimulus, which effectively overlaps with the time window at which auditory integration of the accompanying words occurs. This temporal pattern suggests that beat and accompanied speech processing co-occurred in the same early time window, and it allowed early modulation by gesture, possibly prior to semantic levels of word processing. This result is in line with a previous fMRI study from Hubbard et al. (2009) who suggested that beat gestures are integrated with speech as a part of the visuospatial paralinguistic context. Hubbard et al. found that BOLD responses in multisensory integration areas (i.e., STG/STS) were stronger to speech accompanied by beats rather than non-sense movements. Interestingly, this STS modulation by gestures was absent when gestures were presented on their own, without speech. Our results bring a new temporal dimension to this pattern because they show an early onset of the speech-gesture integration.

It is fair to note that, in our data, the discrimination of the separate effects due to beat gestures as part of the paralinguistic context and those related to simple biological motion perception are difficult to disentangle from a logical point of view. Indeed, when the speaker gestures, there is more biological motion than in the nogesture moments. We must note, however, that there are noticeable differences between our pattern of results and those of previous ERP studies on biological motion perception. For example, Hirai, Fukushima \& Hiraki (2003) showed that when 
participants were presented with biological motion or scrambled motion as a control, differences in the ERPs were found at negative peaks elicited at 200 and $240 \mathrm{~ms}$ post stimulus onset in the occipitotemporal region, which they related to the higher processing of motion stimuli. This is opposite to the positive ERP shift in the gesture condition found in our results at [220-280 ms]. Recently, Krakowski et al. (2011), also found a 200 to $350 \mathrm{~ms}$ negative-going modulation, bilaterally in the posterior middle temporal region when they compared biological motion to scrambled motion conditions, but they also reported an earlier positive-going modulation between 100 and 200ms. However, we found no significant difference between gesture and no gesture conditions in the $[90-180 \mathrm{~ms}]$ time window. Thus, the general timing profile of ERPs to biological motion is quite different from ours and we believe it cannot entirely explain our effects. In addition, please note that despite there may have been differences in the amount of biological motion in our gesture and no-gesture condition, these are by no means optimal to bring out biological motion effects. In our materials, both conditions involved a person moving in a biologically plausible manner. This is in stark contrast with the carefully controlled contrasts used in studies addressing biological motion, where the control stimuli do not involve any biological motion at all. Therefore, we argue that the visually-induced effects seen here relate to effective modulations of speech-related processes.

Indeed, the visual modulation of word-evoked ERPs seen here was effective, as attested by the significant effects detected after subtracting the auditory-only baseline (A) from the signal obtained in AV condition. The very early onset of the modulation (particularly the one at the [10-36ms] time window), is compatible at first sight, with other previous results addressing gestures with ERPs, which reveal that the congruency relationship between speech content and iconic gestures can modulate the auditory ERPs as early as 36 milliseconds post word onset and also at the N1-P2 components (Kelly, Kravitz \& Hopkins, 2004). For now we remain cautious in our interpretation and note only the coincidence of our gesture modulation with the timing of P2, but do not conclude on a direct relationship. What we conclude is that, according to our results, beat gestures seem to affect early sensory and/or phonological processing at pre-semantic levels of analysis by modulating the visuospatial context in which speech is perceived. However, in contrast with iconic gestures, where their descriptive shape, clearly supports additional information to the speech content, the role of beat gestures on the listener's side remains less obvious. 
Based on our results, that reveal effects at pre-semantic stages of speech processing, we hypothesize that beat gestures modulate the general attentional state of the listener during parsing of the utterance. Beats would mostly act as highlighters on important points of the discourse and modulate how verbal information is encoded by attracting listener's attention to specific parts of the speech stream. Our present results certainly conform to the attentional role of beat gestures, because they show early modulations in the auditory signal instead of later semantic levels. In particular, we suggest that beats could modify the form of attention of listener and direct his/her processing resources strategically to relevant aspects of the spoken signal, instead of being regarded as simple non-informative biological movement only. It is important to note that in previous studies each stimulus typically as a very high probability of containing a gesture (one gesture per short clip in some conditions), compared to the natural interweaving of gestures within the speech discourse typical of everyday life spoken language. This may produce high expectation of gestures (compared to real life, where gestures are only a secondary, often unnoticed part of the paralinguistic context), as well as an altered saliency. We used a naturally spoken discourse and yet, even when embedded within a continuous speech stream, beats were salient enough to underline critical points of information. Of course, we cannot forget that in this case the results would be characteristic from a political discourse, where politicians try to attract listener's attention on essential information or concepts.

This attentional hypothesis is indirectly supported by the ample evidence that attention can modulate early phases of the auditory evoked potential and in particular within the P2 time window (see Näätänen, 1982; Hillyard, Hink, Schwent \& Picton, 1973; Picton \& Hillyard, 1974; for seminal studies). Similar modulations have been also observed in speech stimuli, even when using a continuous speech stream, just like in the present study (Astheirmer \& Sanders, 2008), However, because we are talking about the effect of visual events on the auditory evoked potential, multisensory integration processes may also come into play (van Wassenhove, Grant \& Poeppel, 2005; Pilling, 2009). Indeed, Pilling concluded that, in the case of visual lip movements, vision integrates with auditory processing, rather then exerting topdown modulation via attention. The time scale and functional role of beat gestures in speech are very different from those of lip movements, and therefore Pilling's arguments cannot be used as a model in our case. Despite the attentional hypothesis of the modulation within the P2 time window must remain inconclusive for now, it is 
certainly in line with previous results assuming that beats play a concrete role as a highlighter of relevant speech content (McNeill, 1992). For example, previous behavioral experiments have shown that words pronounced with a beat gesture are perceived as more salient than words alone (Krahmer \& Swerts, 2007). Beats are thought to play the same role as head nods or eyebrows which have been shown to produce improvements in comprehension (Krahmer \& Swerts, 2007; Munhall, Jones, Callan, Kuratate \& Vatikiotis-Bateson, 2004). Of course, one potential role of these attentional highlighters would be to facilitate the syntactic parsing of the discourse, as it has been found by Holle et al. (2012), the only study to date addressing beat gestures combined with ERPs as far as we know. Using sentences with ambiguous low frequency syntactic forms, Holle et al. found that beats could modulate the amplitude of the P600 component, a marker of processing cost for syntactic processing. An interesting result in that study was that this modulation was not found when beats were replaced by a moving dot following the same trajectory, or by a pitch change synchronized with the disambiguating noun. Holle et al. proposed that compared to moving dots, beat gestures had a communicative intention determining that visual emphasis increased the plausibility of a low frequency syntactic structure, hinting at a functional role on the listener side too. Note that in our case, we did not manipulate syntactic aspects of the materials, and therefore did not (and could not) measure sentence-level processing. Nevertheless, our results seem to be quite complementary to those of Holle et al.

Interestingly, our results are also consistent with the idea that integration of beat gestures with speech does not involve directing explicit attention to the gestures. Indeed, our participants were merely asked to pay attention to the content of the discourse, not to the gestures, and yet beats led to a positive shift in ERPs. Although many previous studies of gestures did not ask participants to pay attention (for example, Kelly, Creigh and Bartolotti, 2009), their paradigms involved the careful alternation (by trial or by block) of gesture vs. no gesture video clips, with the potential result of altering the natural proportions and cadence of gesture-speech contingencies, and giving away, implicitly, the nature of the manipulation to the observers. Here, the rate and integration of the gestures with the context was just natural, and therefore the paradigm prevented participants to take any special notice, explicit or implicitly, of the gesture manipulation. Also, because beats are even 
simpler than iconic gestures, their integration with speech is strongly likely to be automatic. Actually, both modalities (speech and gesture) may be two components of the same integrated language system. McNeill (1992, p143) suggested that both speech and gestures are in fact aspects of a single process, meaning that all the information available in the speech signal contributes to a general common representation of the content. Later, Kelly, Ozyürek \& Maris (2010) proposed the integrated-systems hypothesis, which postulates that the bidirectional influence of gesture and speech, and the obligatory integration of the two modalities support the claim that they form an integrated system in language comprehension. Willems, Özyürek \& Hagoort (2009) also suggested that the effective co-integration of both modalities depends on the stable semantic relationship between speech and gesture. According to the relative independence between speech and gesture (iconic or pantomime), the STS or the Inferior Frontal Gyrus activations during perception were differently modulated. At this point however, it remains unclear if there are two parallel processes or a common process between both modalities during speech perception.

Finally, it is perhaps important to note that although the statistical analyses highlighted two separate time windows in which gesture modulation of the acoustic signal was significant, the inference about the existence of two dissociable effects (respectively centred on [10-35ms] and [220-280ms]) is not completely granted. An alternative possibility is that beat gestures modulated auditory integration more generally with a positive shift in ERPs at early/sensory levels (Pilling, 2009), but due to the noise present in the signal, sensitivity to ERP differences reached significance at two separate time windows. Wu \& Coulson (2010) used a similar approach with segments of continuous speech and found that when gesture was visible, ERPs were effectively more positive from around $200 \mathrm{~ms}$ until the end of the epoch, at $800 \mathrm{~ms}$. In contrast with the present results, $\mathrm{Wu}$ and Coulson used iconic gestures, which maybe the reason why they found ERP modulations lasting up until semantic processing stages. Indeed, these authors hypothesized that bringing relevant information, iconic gestures allowed synchronized words to be integrated more readily, affecting the whole ERP signal until at least $800 \mathrm{~ms}$. In light of the timing of our effects in the present study, which were restricted within $200 \mathrm{~ms}$ and $280 \mathrm{~ms}$ approximately, we find it unlikely that they encompassed semantic levels of 
processing, indexed by the N400 component (e.g., Wu \& Coulson, 2007). This difference in the time course of the neural correlates of beat gestures (compared to iconic) is consistent with the idea that beats do not trigger speech comprehension in itself, in the way iconic gestures used in past studies may do. However, there are many methodological variations that bar a direct interpretation of the differences between studies. For example here we used different categories of words and not solely nouns. Nevertheless, in terms of topographical distributions, Wu and Coulson also reported the largest effects of gesture at central and centro-parietal sites. We found for our later time window an effect of gesture greater at parietal sites and the topographical distribution also showed greatest differences at centro-parietal sites.

\section{5- CONCLUSION}

Beat gestures modulate the word-evoked ERP correlates in auditory processing during naturalistic conditions of speech perception. This modulation took place at early moments of auditory speech analysis, and effectively affected its processing. Due to its early onset, and synchronization with the gesture, we interpret this modulation as an attentional highlighter affecting a sensory or phonological stage of processing. According to previous results, these attentional highlighters might, later on, be the basis to support the correct or intended syntactic parsing of the sentence. Further behavioral investigations are needed to test whether these effects reflect functional modulation of general attentional state of the listener.

\section{6- REFERENCES}

Astheimer, L. B., \& Sanders, L. D. (2009). Listeners modulate temporally selective attention during natural speech processing. Biological Psychology, 80(1), 23-34.

Casasanto, D., \& Jasmin, K. (2010). Good and bad in the hands of politicians: Spontaneous gestures during positive and negative speech. PloS One, 5(7), e11805. 
Colin, C., Radeau, M., Soquet, A., Demolin, D., Colin, F., \& Deltenre, P. (2002). Mismatch negativity evoked by the McGurk-MacDonald effect: A phonetic representation within short-term memory. Clinical Neurophysiology: Official Journal of the International Federation of Clinical Neurophysiology, 113(4), 495-506.

Gratton, G., \& Coles, M. G. H. (1989). Generalization and evaluation of eye-movement correction procedures. Journal of Psychophysiology, 3, 14-16.

Guthrie, D., \& Buchwald, J. S. (1991). Significance testing of difference potentials. Psychophysiology, 28(2), 240-244.

Hillyard, S. A., Hink, R. F., Schwent, V. L., \& Picton, T. W. (1973). Electrical signs of selective attention in the human brain. Science (New York, N.Y.), 182(4108), 177-180.

Hirai, M., Fukushima, H., \& Hiraki, K. (2003). An event-related potentials study of biological motion perception in humans. Neuroscience Letters, 344(1), 41-44.

Holle, H., Obermeier, C., Schmidt-Kassow, M., Friederici, A. D., Ward, J., \& Gunter, T. C. (2012). Gesture facilitates the syntactic analysis of speech. Frontiers in Psychology, 3, 74.

Hubbard, A. L., Wilson, S. M., Callan, D. E., \& Dapretto, M. (2009). Giving speech a hand: Gesture modulates activity in auditory cortex during speech perception. Human Brain Mapping, 30(3), 1028-1037.

Kelly, S. D., Creigh, P., \& Bartolotti, J. (2010). Integrating speech and iconic gestures in a stroop-like task: Evidence for automatic processing. Journal of Cognitive Neuroscience, 22(4), 683-694.

Kelly, S. D., Kravitz, C., \& Hopkins, M. (2004). Neural correlates of bimodal speech and gesture comprehension. Brain and Language, 89(1), 253-260.

Kelly, S. D., Ozyurek, A., \& Maris, E. (2010). Two sides of the same coin: Speech and gesture mutually interact to enhance comprehension. Psychological Science, 21(2), 260-267. 
Krahmer, E., \& Swerts, M. (2007). The effects of visual beats on prosodic prominence: Acoustic analyses, auditory perception and visual perception. Journal of Memory and Language, 57(3), 396-414.

Krakowski, A. I., Ross, L. A., Snyder, A. C., Sehatpour, P., Kelly, S. P., \& Foxe, J. J. (2011). The neurophysiology of human biological motion processing: A high-density electrical mapping study. Neurolmage, 56(1), 373-383.

McGurk, H., \& MacDonald, J. (1976). Hearing lips and seeing voices. Nature, 264, 746-748.

McNeill D. (1992). Hand and mind: What gestures reveal about thought. Chicago: University of Chicago Press.

Munhall, K. G., Jones, J. A., Callan, D. E., Kuratate, T., \& Vatikiotis-Bateson, E. (2004). Visual prosody and speech intelligibility: Head movement improves auditory speech perception. Psychological Science, 15(2), 133-137.

Naatanen, R. (2001). The perception of speech sounds by the human brain as reflected by the mismatch negativity (MMN) and its magnetic equivalent (MMNm). Psychophysiology, 38(1), 121.

Naatanen, R., Lehtokoski, A., Lennes, M., Cheour, M., Huotilainen, M., livonen, A., Alho, K. (1997). Language-specific phoneme representations revealed by electric and magnetic brain responses. Nature, 385(6615), 432-434.

Naatanen, R. (1982). Processing negativity: An evoked-potential reflection of selective attention. Psychological Bulletin, 92(3), 605-640.

Picton, T. W., \& Hillyard, S. A. (1974). Human auditory evoked potentials. II. effects of attention. Electroencephalography and Clinical Neurophysiology, 36(2), 191-199.

Pilling, M. (2009). Auditory event-related potentials (ERPs) in audiovisual speech perception. Journal of Speech, Language, and Hearing Research : JSLHR, 52(4), 1073-1081 
Stekelenburg, J. J., \& Vroomen, J. (2007). Neural correlates of multisensory integration of ecologically valid audiovisual events. Journal of Cognitive Neuroscience, 19(12), 1964-1973.

Sumby, W., \& Pollack, I. (1954). Visual contribution to speech intelligibility in noise. Journal of the Acoustical Society of America, 26(2), 212-215.

van Wassenhove, V., Grant, K. W., \& Poeppel, D. (2005). Visual speech speeds up the neural processing of auditory speech. Proceedings of the National Academy of Sciences of the United States of America, 102(4), 1181-1186.

Willems, R. M., Ozyurek, A., \& Hagoort, P. (2009). Differential roles for left inferior frontal and superior temporal cortex in multimodal integration of action and language. Neurolmage, 47(4), $1992-$ 2004.

Wu, Y. C., \& Coulson, S. (2007). Iconic gestures prime related concepts: An ERP study. Psychonomic Bulletin \& Review, 14(1), 57-63.

Wu, Y. C., \& Coulson, S. (2010). Gestures modulate speech processing early in utterances. Neuroreport, 21(7), 522-526. 